\title{
Nouvelles perspectives d'amélioration génétique de la prolificité des truies
}

Le développement de la voie hyperprolifique dans les races Large White et Landrace Français et l'utilisation des races prolifiques chinoises ont permis d'accroître de façon notable la prolificité des truies ces dernières années. Néanmoins, l'efficacité d'une sélection directe sur la taille de la portée à la naissance reste limitée par la faible héritabilité de ce caractère (environ 0,10 ) et son expression tardive dans un seul sexe. L'utilisation de races prolifiques chinoises comme la Meishan est quant à elle rendue difficile par leurs faibles performances de croissance et d'engraissement. Les travaux menés ces dernières années visent à repousser ces limites par la recherche de critères de sélection plus efficaces et l'utilisation spécifique des régions intéressantes du génome des races prolifiques chinoises. Ce texte décrit les deux principaux axes de recherche développés à l'INRA dans ce domaine, à savoir le contrôle de la survie prénatale et la recherche de gènes individuels expliquant les écarts de performances entre les races Meishan et Large White.

\section{1 / Sélection sur les composantes de la taille de la portée}

La taille de la portée à la naissance est le résultat d'une série d'événements qui vont de la maturation des gamètes jusqu'à la naissance de porcelets viables : ovulation, fertilisation, développement embryonnaire et fœtal. Le taux de fertilisation est en général proche de $100 \%$ chez le porc et contribue peu aux variations de la prolificité des truies. Celles-ci dépendent donc essentiellement des variations de taux d'ovulation et de survie des embryons ou des fotus.

Le taux d'ovulation (TO) est nettement plus héritable que la taille de la portée à la naissance $(\mathrm{TPN})\left(\mathrm{h}^{2}=0,30\right.$ contre 0,$10 ;$ Blasco et al 1993) et peut aisément être augmenté par sélection, comme l'ont montré deux expériences de sélection réalisées au Nebraska (Lamberson et al 1991) et en France (Bidanel et al 1996a). Mesurable dès la puberté, le taux d'ovulation pourrait donc présenter un intérêt en tant que critère indirect de sélection pour la prolificité. Malheureusement, l'accroissement de TO a été accompagné, dans les deux expériences, par une détérioration de la survie prénatale (SP) et, au final, par une amélioration de TPN nettement inférieure aux valeurs prédites.

Réalisée avec succès chez la souris (Bradford 1969), une sélection sur la survie prénatale n'a été expérimentée que très récemment chez le porc (Bidanel et al 1996a). Les résultats de cette expérimentation réalisée à l'INRA mettent en évidence l'existence d'une variabilité génétique de SP. Son héritabilité n'est toutefois pas supérieure à celle de TPN, de sorte que l'efficacité d'une sélection sur SP n'est pas supérieure à celle d'une sélection directe sur TPN.

Partant du constat qu'une sélection sur TPN se traduisait par une détérioration de la survie prénatale, Johnson et al (1984) ont émis l'hypothèse qu'une sélection sur un indice combinant TO et SP pouvait, dans certaines situations, s'avérer plus efficace qu'une sélection directe sur TPN. La vérification de cette hypothèse a fait l'objet de travaux théoriques et expérimentaux chez la souris et le porc. Une des difficultés à résoudre consistait à modéliser les relations non linéaires existant entre TO, SP et TPN de facon à pouvoir prédire de façon correcte les réponses à une sélection sur ces caractères. Après avoir proposé deux modèles permettant de décrire de façon satisfaisante ces liaisons, Pérez-Enciso et al (1996) ont comparé par simulation l'efficacité d'une sélection sur indice I(TO,SP) à celle d'une sélection directe sur TPN. Leurs résultats montrent clairement qu'une sélection sur indice présente en général peu d'intérêt par rapport à une sélection directe sur TP. Ces conclusions sont en accord avec les résultats expérimentaux obtenus au Nebraska (Casey et al 1994).

Notant que l'augmentation de TO dans l'expérience de sélection du Nebraska s'accompagnait d'un accroissement de la mortalité fœetale, Christenson et al (1987) ont quant à eux proposé de sélectionner les femelles sur une mesure indirecte de leur efficacité utérine (EU = nombre maximum de produits qu'une femelle est apte à conserver jusqu'au terme de la gestation), à savoir le nombre de produits nés d'une femelle ayant subi une " hémihystéro-ovariectomie ». L'ablation d'une corne utérine et d'un ovaire réduit l'espace utérin disponible tout en maintenant un taux d'ovulation comparable à celui d'une femelle non opérée, et permet ainsi de mesurer EU. Des expériences de sélection sur EU chez la souris et la lapine ont permis d'accroître de façon significative EU et TPN chez des femelles non opérées, mais sans démontrer la supériorité de cette stratégie sur une sélection directe sur TPN (Kirby et Nielsen 1993, Argente et al 1997).

De même, une augmentation significative de TPN est obtenue par Robison et al (1994) dans une expérience de sélection sur le taux circulant de testostérone, mais sans comparaison de l'efficacité relative de ce critère indirect par rapport à une sélection directe sur TPN.
J.P. BIDANEL

INRA Station

de Génétique

quantitative

et appliquée,

78352 Jouy-en-Josas

Cedex 
Tableau 1. Principaux gènes majeurs et locus à effets quantitatifs affectant la taille de la portée et ses composantes localisés chez le porc.

\begin{tabular}{|c|l|l|l|}
\hline Chromosome & \multicolumn{1}{|c|}{ Caractères } & \multicolumn{1}{|c|}{ Localisation probable } & \multicolumn{1}{c|}{ Référence } \\
\hline 1 & Taille de la portée & Région du locus ESR & Rothschild et al 1996 \\
6 & Taille de la portée & Locus du groupe sanguin H & Jensen et al 1968 \\
7 & Taux d'ovulation & SLA-S0102 & Bidanel et al 1998 \\
8 & Taux d'ovulation & Sw790 & Rahtje et al 1997 \\
& Nombre d'embryons & S0225-Sw1551 & Bidanel et al 1998 \\
17 & Taille de la portée & Récepteur à la mélatonine & Ollivier et al 1997 \\
\hline
\end{tabular}

Dans l'état actuel de nos connaissances, aucun des critères indirects de sélection pour la prolificité étudiés jusqu'à présent n'apparaît donc clairement plus efficace qu'une sélection directe sur TPN.

\section{2 / Mise en évidence et sélection de gènes à effets individuels importants}

Le développement récent de cartes génétiques couvrant de façon exhaustive le génome du porc (Ellegren et al 1994, Archibald et al 1995, Rohrer et al 1994 et 1996) permet désormais d'envisager une recherche systématique des gènes à effets individuels importants sur les caractères d'intérêt économique (ces gènes sont souvent désignés par le sigle QTL, abréviation du terme anglais « Quantitative Trait Locus »). Les principes généraux d'une telle recherche ont été présentés dans un article précédent (Bidanel et al 1996b). Des programmes de recherche de QTL ont été mis en place ces dernières années dans plusieurs pays, le plus souvent dans des populations issues de croisements entre des races ou espèces phénotypiquement très différentes (Large White et Meishan, Large White ou Piétrain et Sanglier). Ainsi, l'INRA développe depuis 1991 un programme important de recherche de QTL dans une population F2 Meishan x Large White. Des programmes de recherche de QTL sur des populations en ségrégation ont également été menés en utilisant notamment des expériences de sélection et/ou le typage d'individus extrêmes (Ollivier et al 1997).
Les premiers résultats de ces expérimentations voient le jour depuis trois ans. Si l'on inclut les résultats anciens relatifs aux effets du groupe sanguin $\mathrm{H}$ (Jensen et al 1968), cinq régions chromosomiques affectant la taille de la portée ou ses composantes ont actuellement été mises en évidence sur les chromosomes 1 , 6, 7, 8 et 17 (tableau 1). Les effets de ces QTL sont relativement importants, avec des effets de substitution allélique variant entre 0,75 et 3 corps jaunes, embryons ou porcelets. On peut en particulier noter que la région du chromosome 8 concernée est la région homologue de celle du chromosome 6 ovin où a été localisé le locus $\mathrm{Fec}^{\mathrm{B}}$ (ou «Booroola ») qui présente un effet majeur sur le taux d'ovulation dans cette espèce.

L'intérêt d'une prise en compte de l'information sur des gènes à effets individuels dans les schémas d'amélioration génétique a fait l'objet de nombreux travaux théoriques ces dernières années. On peut notamment envisager d'introduire un allèle favorable dans une population qui en est dépourvue (on parle alors d'introgression) ou réaliser une sélection lorsqu'il y a ségrégation au QTL. L’introgression ou la sélection se fera directement sur le génotype des individus au QTL lorsque celui-ci est connu ou à partir de leur génotype pour des marqueurs flanquants dans le cas, en pratique le plus fréquent, où le génotype au QTL n'est pas accessible. On parle alors d'introgression ou de sélection assistées par marqueurs (Bidanel et al 1996b). En pratique l'intérêt d'une prise en compte de l'information sur des QTL dans un programme d'amélioration génétique sera fonction de nombreux facteurs comme le polymorphisme aux QTL dans les populations concernées, l'importance de leurs effets sur les différents caractères d'intérêt économique et la fiabilité avec laquelle le génotype des animaux aux QTL est estimé. Cet intérêt est également d'autant plus grand que de l'efficacité des méthodes classiques de sélection est réduite. En particulier, comme l'illustrent les résultats du tableau 2, l'intérêt potentiel de la prise en compte de l'information sur des QTL est plus élevé pour les caractères à faible héritabilité tels que la taille de la portée chez le porc. Des études complémentaires restent toutefois nécessaires afin de comparer l'efficacité de ces

Tableau 2. Proportion $R$ de la variance génétique additive expliquée par un QTL nécessaire pour accroître de $25 \%$ l'efficacité d'une sélection utilisant l'information sur le QTL par rapport à une sélection sur performances uniquement (d'après Smith 1967).

\begin{tabular}{|l|c|c|c|}
\hline \multicolumn{1}{|c|}{ Caractère } & $\begin{array}{c}\text { Gain moyen } \\
\text { quotidien }\end{array}$ & $\begin{array}{c}\text { Surface du muscle } \\
\text { long dorsal }\end{array}$ & Taille de la portée \\
\hline Héritabilité & 0,4 & 0,4 & 0,1 \\
\hline $\begin{array}{l}\text { Méthode de sélection } \\
\text { sur performances }\end{array}$ & individuelle & $\begin{array}{c}\text { moyenne de 3 } \\
\text { collatéraux de portée }\end{array}$ & $\begin{array}{c}\text { moyenne des 2 premières } \\
\text { portées de la mère }\end{array}$ \\
\hline Proportion donnant un gain de 25 & de réponse à la sélection \\
\hline Sélection sur le QTL & 0,62 & 0,17 & 0,07 \\
\hline Sélection sur performances + QTL & 0,20 & 0,09 & 0,02 \\
\hline
\end{tabular}


méthodes par rapport à une sélection utilisant des indices de type "BLUP - modèle animal ", nettement plus efficace que les méthodes de sélection sur performances utilisées dans le tableau 2.

\section{Références}

Archibald A.L. et al, 1995. The PiGMaP consortium linkage map of the pig (Sus scrofa). Mammalian Genome, 6, 157-175.

Argente M.J., Santacreu M.A., Climent A., Bolet G., Blasco A., 1997. Divergent selection for uterine capacity in rabbits. J. Anim. Sci., 75, 2350-2354.

Bidanel J.P., Blasco A., Dando P., Gogué J., Lagant H., 1996a. Résultats de quatre générations de sélection pour le taux d'ovulation et la survie prénatale chez des porcs de race Large White. Journées Rech. Porcine en France, 28, 1-8.

Bidanel J.P., Bonneau M., Chardon P., Elsen J.M., Gellin J., Le Roy P., Milan D., Ollivier L., 1996b. Etablissement et utilisation de la carte génétique porcine. INRA Prod. Anim., 9, 299-310.

Bidanel J.P., Milan D., Chevalet C., Woloszyn N., Bourgeois F., Caritez J.C., Gruand J., Le Roy P., Bonneau M., Lefaucheur L., Mourot J., Prunier A., Désautés C., Mormède P., Renard C., Vaiman M., Robic A., Gellin J., Ollivier L., 1998. Détection de locus à effets quantitatifs dans le croisement entre les races porcines Large White et Meishan. Dispositif expérimental et premiers résultats. Journées Rech. Porcine en France, 30, 117-125.

Blasco A., Bidanel J.P., Bolet G., Haley C.S., Santacreu M.A., 1993. The genetics of prenatal survival of pigs and rabbits : a review. Livest. Prod. Sci., 37, 121.

Bradford G.E., 1969. Genetic control of ovulation rate and embryo survival in mice. I - Response to selection. Genetics, 61, 905-921.

Casey D., Rathje T.A., Johnson R.K., 1994. Second thoughts on selection for components of reproduction in swine. In : Proc. 5th World Congress on Genetics Applied to Livestock Production, 17, 315-318.

Christenson R.K., Leymaster K.A., Young L.D., 1987. Justification of unilateral hysterectomy-ovariectomy as a model to evaluate uterine capacity in swine. J. Anim. Sci., 65, 738-744.
Ellegren H., Chowdhary B., Johansson M., Andersson L., 1994. A primary linkage map of the porcine genome reveals a low rate of recombination. Genetics, 137, 1089-1100.

Jensen E.L., Smith C., Baker L.N., Cox D.F., 1968. Quantitative studies on blood group and serum protein systems in pigs. II. - Effects on production and reproduction. J. Anim. Sci., 27, 856-862.

Johnson R.K., Zimmerman D.R., Kittok R.J., 1984. Selection for components of reproduction in swine. Livest. Prod. Sci., 11, 541-558.

Kirby Y.K., Nielsen M.K., 1993. Alternative methods of selection for litter size in mice : III. Response to 21 generations of selection. J. Anim. Sci., 71, 571-578.

Lamberson W.R., Johnson R.K., Zimmerman D.R., Long T.E., 1991. Direct responses to selection for increased litter size decreased age at puberty, or random selection following selection for ovulation rate in swine. J. Anim. Sci., 69, 3129-3143.

Ollivier L., Messer L.A., Rothschild M.F., Legault C., 1997. The use of selection experiments for detecting quantitative trait loci. Genetical Research, 69, 227232.

Pérez-Enciso M., Bidanel J.P., Baquedano I., Noguera J.L., 1996. A comparison of alternative genetic models for litter size in pigs. Anim. Sci., 63, 255-264.

Rathje T.A., Rohrer G.A., Johnson R.K., 1997. Evidence for quantitative trait loci affecting ovulation rate in pigs. J. Anim. Sci., 75, 1486-1494.

Robison O.W., Lubritz D., Johnson B., 1994. Realized heritability estimates in boars divergently selected for testosterone levels. J. Anim. Breed. Genet., 111, $35-42$.

Rohrer G.A., Alexander L.J., Keele J.W., Smith T.P.L., Beattie C.W., 1994. A microsatellite linkage map of the porcine genome. Genetics, 136, 231-245.

Rohrer G.A., Alexander L.J., Hu Z., Smith T.P.L., Keele J.W., Beattie C.W., 1996. A comprehensive map of the porcine genome. Genome Res., 6, 371391.

Rothschild M.F., Jacobson C., Vaske D.A., Tuggle C., Wang L., Short T., Eckardt G., Sasaki S., Vincent A., McLaren D.G., Souwthwood O., van der Steen H. Mileham A., Plastow, G., 1996. The Estrogen Receptor locus is associated with a major gene influencing litter size in pigs. Proc. Nat. Acad. Sci. (USA), 93, 201-205.

Smith C., 1967. Improvement of metric traits through specific genetic loci. Anim. Prod., 9, 349-358.

\section{Contrôle du taux d'ovulation chez la truie : l'apport des modèles hyperprolifiques}

Parmi les nombreuses composantes de la productivité numérique (nombre de porcelets sevrés par truie et par an), la prolificité, ou taille de la portée à la naissance, est devenue un critère prioritaire de sélection. La prolificité dépend de deux facteurs principaux : d'une part le nombre d'ovulations (qui en fixe la limite supérieure) et d'autre part la mortalité embryonnaire et fœtale. Une prolificité élevée peut donc être obtenue via une augmentation du nombre d'ovulations sans réduction de la mortalité embryonnaire (cas de la

\section{M.A. DRIANCOURT, F. MARTINAT-BOTTÉ, M. TERQUI}

INRA Physiologie de la reproduction des mammifères domestiques, 37380 Nouzilly 